\title{
Le «médecin croyant» et le théologien genevois
}

\section{Une lettre écrite en 1802 par M.F. R. Buisson à Pierre Picot}

par Jean Starobinski

\section{RÉSUMé}

Le médecin Matthieu-François-Régis Buisson (1776-1804) relate dans cette lettre à Pierre Picot, professeur de théologie à l'Académie de Genève, le climat tendu dans lequel s'est déroulée la soutenance de sa thèse à la Faculté de médecine de Paris. L'auteur prône une sorte de sainte alliance des médecins croyants et des théologiens contre le parti alors dominant à l'Ecole de médecine, où règnent en maîtres le courant idéologique issu de l'Encyclopédie, l'anticléricalisme de Pinel et le monisme matérialiste de Cabanis.

\section{Introduction}

Le document que nous présentons est une lettre signée, adressée de Paris par le médecin Matthieu-François-Régis Buisson (Lyon 1776-Paris, octobre 1804) au professeur genevois Pierre Picot-Trembley (1745-1822). Cette lettre est conservée au département des manuscrits de la Bibliothèque Publique et Universitaire de Genève.

Pierre Picot fut pasteur à Satigny, puis professeur honoraire d'Histoire ecclésiastique à l'Académie de Genève (1787-1795); il succéda, en 1795, à Antoine Maurice, au titre de professeur de Théologie. Il exerce la fonction de Recteur de l'Académie, de 1790 à 1792. Il quitte l'enseignement en 1817. Il s'était vu confier les fonctions de doyen de sa Faculté ${ }^{1}$.

Notre document l'indique bien: c'est Pierre Picot qui prit l'initiative d'écrire à Buisson, après avoir lu un «extrait» du livre que celui-ci venait de publier (en septembre 1802) ${ }^{2}$. On peut imaginer un intermédiaire en la personne du docteur Jean-Pierre Maunoir (1768-1861)³, qui avait été, comme Bichat, un élève de Desault, et qui entretenait des relations amicales avec de nombreuses personnalités médicales parisiennes, notamment avec Jean-Noël Hallé (1754-1822). 
Régis Buisson, né à Lyon, était le cousin de Xavier Bichat. Les événements sanglants qui bouleversèrent cette ville en 1793 les impressionnèrent l'un et l'autre. A Paris, Buisson fut le disciple et le collaborateur de son cousin. Après la mort de celui-ci, il acheva, avec P.-J. Roux, l'Anatomie descriptive qui était restée à l'état d'ébauche. Du vivant même de Bichat, Buisson avait entrepris la critique de la première partie des Recherches physiologiques sur la vie et la mort. Cette critique prit forme dans l'ouvrage De la divison la plus naturelle des phénomènes physiologiques considérés dans l'homme. C'est la thèse de doctorat que Buisson soutint le 7 septembre 1802 ; elle fut imprimée chez Feugueray et vendue chez Brosson. Le livre était augmenté d'un «précis historique sur M. F. X. Bichat». Il y est rappelé que «la plénitude» de la confiance de Bichat «fut toujours réservée à sa famille». Et Buisson ajoute: «Nous seuls connoissions son ame tout entière...» Buisson allait mourir en octobre 1804, à 28 ans.

Le cousin de Bichat n'aura donc laissé qu'un seul ouvrage, où il corrige d'un point de vue spiritualiste la distinction devenue rapidement fameuse entre la vie animale et la vie organique. Buisson résume d'entrée de jeu ses arguments :

«Puisque l'homme diffère des animaux par des attributs essentiels, c'est partir d'un faux principe que de le confondre avec eux, et d'établir, pour terme unique de comparaison dans la nature organisée, des animaux et des végétaux.

Puisqu'il est chez l'animal un ordre de fonctions nutritives que le végétal ne présente point, c'est une erreur de ne distinguer ces deux êtres que par l'appareil sensitif et locomoteur dont l'animal est pourvu.» ${ }^{4}$

L'affaire essentielle, pour Buisson, est d'établir les bases d'une meilleure division. L'homme, contrairement à l'animal, est capable d'une véritable vie active, guidée par l'intelligence, qui est un principe immatériel. Buisson fait sienne la définition formulée par Louis de Bonald, le théoricien de la contrerévolution monarchiste et catholique: «L'homme est une intelligence servie par des organes». ${ }^{5}$ Bonald est l'autorité intellectuelle dont Buisson se réclame au long de son ouvrage. Nous apprenons, par la lettre à Pierre Picot, que le jeune médecin et le philosophe sont unis par des liens d'amitié, qu'ils ont de fréquents entretiens, et qu'ils considèrent leurs travaux comme parfaitement complémentaires.

Buisson s'empresse donc de faire parvenir à Pierre Picot deux ouvrages récents de Bonald: l'Essai analytique sur les lois naturelles de l'ordre social (1800) et Du divorce considéré au dix-neuvième siècle (1801). Après la mort de Buisson, Louis de Bonald ne manquera pas de lui rendre hommage, tout en 
reprenant quelques-unes de ses idées. Dans les Recherches philosophiques sur les premiers objets des connaissances morales (1817), on lit, dans une note du chapitre IV (De la physiologie), un éloge de Buisson. Il «aurait marché sur les traces» de «Descartes, Malebranche, Haller, Ch. Bonnet, Stahl surtout»...

«Au sortir de l'école, il se livra à un travail forcé, et périt bientôt victime de ses devoirs. Cet excellent jeune homme réunissait, au talent le plus décidé pour sa profession, toutes les qualités aimables, toutes les vertus religieuses et civiles $[\ldots]$ Son ouvrage $[\ldots]$ présente les vues les plus saines sur les questions que nous traitons ici, et sur l'accord de la physiologie et de la morale.» ${ }^{6}$

La place de Buisson est restée assez modeste dans la littérature médicale du $\mathrm{XIX}^{\mathrm{e}}$ siècle. Dezeimeris lui consacre une notice, dont Beaugrand extrait un passage pour l'article Buisson du Dictionnaire de Dechambre ${ }^{7}$. Le docteur Louis Cerise, qui fit paraître une édition commentée des Recherches de Bichat, partageait les vues de Buisson, qu'il cite amplement ${ }^{8}$. Mais Buisson a été lu surtout par les philosophes. Il est cité par Maine de Biran ${ }^{9}$, par Ravaisson ${ }^{10} \ldots$ Rien d'étonnant à cela : le livre de Buisson est de nature toute spéculative. Ce n'est pas l'ouvrage d'un expérimentateur, ni même d'un observateur (la première partie des Recherches de Bichat ne l'était pas non plus). Il cite les philosophes et les poètes anciens (Cicéron, Quintilien, Virgile), au même titre que les philosophes, les naturalistes et les médecins modernes: Stahl, Bonnet, Haller, Pinel, Hallé et, bien sûr, son proche et intime adversaire Bichat. Dans le livre de Buisson, comme dans la lettre qu'on va lire, on remarquera le rôle important de Stahl. On le sait, Bichat était influencé par la tradition montpelliéraine plus que ne l'étaient des collègues parisiens. Sur ce point, son cousin lui ressemble. Mais tandis que la plupart de ses contemporains se défient de la «métaphysique», Buisson affronte résolument la vieille question des rapports de l'âme et du corps. Si l'âme, selon Buisson, est responsable de la «vie active», il ne croit pas, comme Stahl, qu'elle veille immédiatement, et de façon obscure, à l'accomplissement des fonctions organiques. Le problème restera débattu dans la première partie du dix-neuvième siècle, et la question du «duodynamisme» fera couler encore beaucoup d'encre en milieu académique ${ }^{11}$.

Buisson, admirateur et disciple de Bonald, se sent en butte à l'hostilité de la majorité de ses maîtres et de ses condisciples de l'Ecole de médecine. Cette école est encore celle que Fourcroy a mise en place, et où prédomine l'influence de l'Encyclopédie, d'Helvétius, de d'Holbach, des «idéologues». L'anticléricalisme de Pinel, le monisme matérialiste de Cabanis donnent le 
ton ${ }^{12}$. Membre fondateur de la Congrégation, où il côtoie G. L. Bayle, le jeune Laennec, Fizeau, Savary, notre auteur n'est pas un isolé ${ }^{13}$. Il appartient à un groupe d'opposants qui s'est donné une tâche polémique: ce parti triomphera sous la Restauration et obtiendra le renvoi de Pinel. Mais, on le sait, la Faculté de médecine avait été et restera un champ de bataille idéologique. La lettre de Buisson à Picot, relatant le climat tendu de la soutenance du 7 septembre 1802, fait penser à l'atmosphère batailleuse dans laquelle se déroula, en cette même année, la soutenance de Gaspard Laurent Bayle. Quand la faveur royale, après 1815, favorisa les «médecins croyants», ce fut au tour de Broussais et de ses élèves de manifester leur opposition...

On comprend dès lors que dans sa lettre empressée au protestant Pierre Picot, le catholique Buisson oublie la vieille dispute entre confessions, dans l'intérêt d'un large ralliement des défenseurs du christianisme. Une sorte de sainte alliance des médecins et des théologiens doit être établie sans tarder, la priorité étant de faire front contre ceux qu'il nomme «les ennemis de la vérité».

\section{Le texte de la lettre de M. F. R. Buisson à Pierre Picot}

L'auteur de cette lettre est mort en 1804 universellement regretté ${ }^{1}$.

Paris, 12 Octobre 1802

Je m'empresse, Monsieur, de répondre au désir que vous me témoignez de manière si flatteuse et si honorable pour moi. Mon ouvrage a pour but principal la défense d'une de ces vérités fondamentales sur lesquelles reposent le bonheur des hommes et leur espérance. Dès lors il appartient de droit à tous ceux qui se proposent la même fin. Veuillez donc bien l'accepter à ce titre comme un témoignage de mon estime et de ma reconnaissance. Je n'aurais pas attendu la demande que vous m'en faites, si j'avais eu l'avantage de vous connoître.

Vous paroissez voir avec quelque étonnement, Monsieur, qu'un Médecin se voit chargé de plaider la cause de la dignité de l'homme et malheureusement on est fondé aujourd'hui à s'en étonner. C'est principalement par la médecine qu'a porté cette funeste épidémie que vous déplorez avec tant de raison. Presque tous ceux qui cultivent et qui enseignent aujourd'hui l'art de guérir, sont atteints sous ce rapport d'une maladie mortelle et ce qu'il y a de plus fâcheux, c'est qu'elle ne cesse point d'être contagieuse. L'Ecole de Paris dans le sein de laquelle j'ai été formé, en est spécialement infectée, et tel est le délire général que depuis longtemps on y regarde comme un axiôme cette ridicule assertion qu'il est impossible dans l'état actuel de nos connoissances d'être à la fois Médecin et croyant. On oublie que tous les hommes illustres dans notre art ont réuni constamment ces deux qualités, que les ouvrages des Médecins croyants sont les seules sources où l'on puise encore pour se former, et qu'on ne fait absolument que développer des idées dont ils ont donné les premiers apperçus. L'état actuel de nos connoissances est un de ces propos 
vagues qu'on met sans cesse en avant, avec lequel on croit répondre à tout, dont la multitude se paye aveuglément et que les maîtres eux-mêmes prononcent le plus souvent sans se rendre compte de sa valeur. Il y auroit bien à dire là dessus si on réfléchissait un peu et une grande partie de l'échaffaudage scientifique glacé dont on nous fait peur aujourd'hui s'écrouleroit bientôt si on faisoit briller sur lui pendant quelques moments le flambeau d'une raison saine et exempte des préjugés philosophiques.

Disons-le cependant et publions-le avec cette satisfaction vive qu'inspire l'amour de la vérité, l'Ecole de Paris renferme encore un bon nombre d'esprits justes et de cœurs droits qui résistent courageusement à la dépravation générale. Les efforts des maîtres n'ont pas entraîné tous les disciples et ce qu'il y a de plus singulier, c'est que ce sont précisément des imbécilles, des esprits foibles qui soutiennent presque seuls l'honneur de l'Ecole et à qui elle est obligée de distribuer presqu'exclusivement les récompenses publiques qu'elle donne à la fin de chaque année. Je suis un des premiers à qui cette honorable qualification d'imbécille ait été appliquée soit par les Professeurs, soit par la foule de mes condisciples. On me regardoit même comme le chef du parti fanatique, comme le maniaque par excellence. Le sens des mots étant une fois changé, j'ai trouvé ces titres tellement flatteurs que mon unique ambition a été de les mériter, et si j'ai redoublé de travail, ç'a toujours été dans la vue de prouver contre le prétendu axiôme qu'on pouvait être Médecin et croyant. La Providence a daigné me favoriser.

L'année dernière je remportais le premier prix dans un concours public, ce qui me donna plus de facilité pour mettre ouvertement au jour les principes qui me dirigent. J'entrepris alors l'ouvrage que je viens de publier et qui m'a servi de thèse de réception comme Médecin. C'était la première fois qu'on parloit un tel langage dans l'Ecole et qu'on se présentoit pour soutenir des idées diamétralement opposées au matérialisme. On s'attendoit à voir une dispute très vive et très animée; je m'y attendois plus que personne d'autant plus que j'avais au nombre de mes examinateurs deux des plus ardents défenseurs de l'Athéisme et de l'animalisme ${ }^{2}$. Plus de cinq cents jeunes gens se rassemblèrent, les uns avec inquiétude sur mon succès qu'ils désiroient vivement, les autres avec l'espérance d'une défaite qu'ils souhaitoient beaucoup. Nous fûmes tous trompés en grande partie. Les Professeurs me traitèrent avec des égards dont on fut généralement surpris; un seul me fit des objections assez spécieuses, et on témoigna par des applaudissements unanimes qu'on étoit satisfait de mes réponses. C'est le 7 septembre dernier que ceci eut lieu.

Pardonnez, Monsieur, si je vous entretiens si longtemps de moi. J'ai cru ces détails nécessaires pour vous mettre à même de juger plus complettement l'esprit dans lequel mon ouvrage est composé. D'ailleurs le zèle que vous montrez pour la défense des vrais principes ne me permet pas de douter que vous n'appreniez avec intérêt ce petit triomphe de la vérité sur l'erreur. Je viens maintenant à l'ouvrage considéré en lui-même.

Vous avez été frappé de ma définition de l'homme. L'extrait où vous l'avez vue a dû vous apprendre que je n'en suis point l'auteur. Je l'ai trouvée dans les écrits d'un homme accoutumé aux plus sublimes considérations métaphysiques et dont la Philosophie uniquement fondée sur les grandes lumières que la Religion fait briller dans l'univers, semble avoir été réservée à notre siècle comme une digue puissante contre le torrent de l'incrédulité. La société particulière et les entretiens de cet ami respectable m’ont été d'une utilité plus grande encore que ses ouvrages. Etranger aux connoissances physiologiques, il ne pouvoit donner aux belles idées qu'il avoit conçues sur l'homme tous les développements dont elles étoient susceptibles; mon travail peut done être regardé comme une suite du sien. Afin que vous puissiez en juger par vous-même, Monsieur, je vous envoye sous la même enveloppe les deux ouvrages de M. de Bonald qui sont le 
plus souvent cités dans le mien ${ }^{3}$. C'est l'auteur lui-même qui vous les offre; il a vu avec la plus vive satisfaction la lettre dont vous m'avez honoré. Ami de tous ceux qui s'opposent aux nouvelles doctrines, il vous prie d'agréer l'assurance de sa plus haute considération.

Vos réflexions, Monsieur, sur les autres êtres auxquels la définition de l'homme peut convenir, sont dignes d'une ame grande et élevée. Malebranche a déjà fait la même supposition, et rien n'empêche de l'admettre ${ }^{4}$. Mais il me semble aussi que rien ne nous y oblige. Nous pouvons raisonner ici sur le plus ou moins de probabilités, mais non sur des preuves, puisque nous n'en avons aucune. Qu'il y ait d'autres mondes peuplés comme le nôtre, que ces mondes soient habités par des Etres intelligents semblables ou supérieurs à l'homme, c'est sur quoi nous ne saurons jamais rien de positif jusqu'au jour de la manifestation générale de toute chose, omnis rei tempus, selon la belle expression de l'Ecclésiaste. Je crois qu'on doit abandonner toute discussion sur cet article qui du reste est fort indifférent à notre bonheur. S'il était possible que nous fissions quelque jour ces grandes découvertes, un nouvel ordre de faits se présenteroit et exigeroit de nouvelles considérations qui ne contrediroient point les anciennes. La définition de l'homme s'étendroit sans aucune difficulté à d'autres êtres, et n'en seroit pas moins toujours celle de l'homme.

Je vois, Monsieur, que vous partagez absolument l'opinion de Stahl sur l'union des deux substances. Comme lui vous attribuez tous les phénomènes organiques à une volonté implicite de l'âme qui a besoin du corps et le conserve par sa présence. Ce n'est pas là le sentiment des Physiologistes modernes et j'avoue que ce n'est pas non plus le mien. Car d'abord attribuer tout ce qui se passe dans le corps à l'action du principe pensant, c'est se mettre dans l'inévitable nécessité d'accorder un principe pensant aux animaux puisque chez eux nous trouvons les mêmes principes organiques que chez nous. En second lieu, quelle idée peut-on se former d'une volonté implicite qui fait mouvoir le cœur et les organes soit digestifs, soit sécrétoires etc. quand tout nous prouve que la digestion, la circulation, les sécrétions etc. s'opèrent d'une manière continue, uniforme, nécessaire, sans que le moi humain y prenne aucune part? Admettre cette volonté implicite, c'est, ce me semble, retomber dans les hypothèses gratuites qu'il est essentiel aujourd'hui d'éviter plus que jamais. Je dis, hypothèses gratuites, car je ne regarde point comme une explication satisfaisante cette espèce d'axiôme sur lequel s'appuye Stahl: Qui veut la fin, veut les moyens. Il est en effet très possible que celui qui veut la fin, ne soit pas maître de tous les moyens et par conséquent ne puisse les vouloir tous directement; que plusieurs de ces moyens soient coordonnés primitivement par une volonté supérieure de manière que la fin soit toujours obtenue. En un mot, volonté, suppose puissance; puissance suggère liberté. Or l'âme n'a évidemment aucune liberté pour suspendre les mouvements du cœur tandis qu'elle en jouit pour arrêter les mouvements du bras. Les contractions du cœur durent encore quelque temps et se font avec régularité après la mort, le cœur étant même séparé de la poitrine. Peut-on dire que ce soit alors le principe pensant qui les détermine?

Disons plutôt qu'il y a chez l'homme deux ordres de phénomènes organiques tout à fait distincts. Les premiers ont pour fin le service de l'intelligence, tels sont ceux des sens, de la voix, de la locomotion. Ils sont toujours soumis à la volonté; eux seuls concourent essentiellement à constituer l'homme. Les seconds ont pour fin la conservation des organes qui font le service de l'intelligence. Ce sont ceux de la digestion, de la respiration, de la circulation, etc. La volonté influe encore indirectement sur plusieurs, mais le plus grand nombre est entièrement soustrait à cette influence dès le moment où l'homme existe, de manière qu'ils s'exécuteront toujours uniformément, en vertu de certaines lois. Ceci demanderoit des développements très étendus. 
J'ai tâché de les donner dans mon ouvrage.

En un mot j'admets avec Stahl que le corps est l'organe de l'âme; je n'admets point avec lui que l'âme soit la cause immédiate de tous les mouvements organiques. Tous ces mouvements s'opèrent médiatement ou immédiatement pour le service de l'intelligence; tous ne sont pas dirigés et coordonnés immédiatement par elle. Ceci ne nous mène pas comme on pourroit le craindre d'abord à l'harmonie préétablie de Leibniz. Car Leibniz considère l'âme et le corps non seulement comme distincts, mais comme isolés, agissant chacun de son côté et se trouvant seulement en rapport constant dans leurs actions. Je vois au contraire l'âme agissant toujours par le moyen du corps et employant à son usage les propriétés vitales dont ce corps jouit pendant un temps que la volonté du Créateur a circonscrit et déterminé.

Je me réjouis beaucoup, Monsieur, d'apprendre que les travaux de votre Académie tendent au même but que les miens. Je vous serois infiniment obligé si vous voulez bien me communiquer quelquefois les principaux résultats de ces travaux. Depuis longtemps les ennemis de la vérité se liguent ensemble pour la combattre; il est temps que ses amis se réunissent de toutes parts pour les défendre.

M. Marignié ${ }^{5}$, directeur du Publiciste ${ }^{6}$, ayant appris que je vous écrivais, m'a chargé de vous offrir ses compliments affectueux.

Je n'oserois décider absolument si aucun lien éloigné ne me rapproche de la famille dont vous me parlez ${ }^{7}$. La mienne est de Lyon où moi-même je suis né. La proximité des lieux peut donner quelque probabilité à cette liaison dont je me trouverois fort honoré.

Agréez, Monsieur, l'expression sincère de mon respect et de ma reconnoissance.

P. S. Vous recevrez par la diligence le paquet que je vous ai annoncé

\section{Buisson}

médecin, rue d'Enfer no 771, à l'entrée de la place St-Michel.

(deux ans après)

Mr Buisson jeune médecin de grande espérance est mort quelque temps après avoir écrit cette lettre.

Monsieur de Bonald dont il est ici parlé est le même qui a figuré ensuite comme l'un des Inspecteurs Généraux de l'Université Impériale ${ }^{8}$. 


\section{Introduction}

1 De la main de Picot. On lira: Borgeaud, Charles, Histoire de l'Université de Genève. L'Académie de Calvin, 1559-1798. Genève, Georg, 1900; L'Académie de Calvin dans l'Université de Napoléon, 1798-1814. Genève, Georg, 1909.

2 Où cet extrait avait-il paru ? La lettre de Pierre Picot à Buisson a-t-elle été conservée ? Une recherche reste à faire.

3 Sur Jean-Pierre Maunoir, cf. Borgeaud, Charles (1909) et les articles d'Albert Th. Franceschetti et de Roger Mayer dans Gesnerus, Vol.32, 1975, Fasc. 1/2, «Aspects historiques de la médecine et des sciences naturelles en Suisse romande.»

4 De la divison $[\ldots$,$] p. 29$.

5 La formule se trouve dans la Préface de Du divorce..., 1801.

6 Dans l'édition de 1853, cette note figure aux pp.137-138.

7 Dictionnaire encyclopédique des sciences médicales, ed. A. Dechambre, tome onzième, Paris, s. d., pp. 303-304.

8 Cf. Bichat, F.X., Recherches physiologiques sur la vie et la mort, nouvelle édition précédée d'une notice [...] et suivie de notes par le docteur Cerise, Paris, 1856, pp. 279-280.

9 Voir en particulier le Mémoire sur la décomposition de la pensée, édité par François Azouvi, au tome III des Euvres, Paris, Vrin, 1988.

10 Ravaisson, Félix, De l'habitude (1838), édité par J. Baruzi, Paris, Alcan.

11 Sur ce débat, on consultera Bouillier, Francisque, Du principe vital et de l'âme pensante, Paris, 1862; deuxième éd. augmentée, 1873. Il n'y est pas fait mention de Buisson.

12 Cf. Ackerknecht, Erwin H., Medicine at the Paris Hospitals, 1794-1848. Johns Hopkins Press, Baltimore, 1967.

13 Cf. Grandmaison G. de, La Congrégation, Paris, 1889. Grandmaison signale que la Congrégation, fondée le 2 février 1801 autour du père jésuite Delpuits, eut Régis Buisson pour premier préfet. Régis Buisson dédie (en latin) son ouvrage à son oncle maternel, Joachim Bichat. Cet oncle, qui prit soin de son éducation («Qui mihi primos reclusit scientiarum fontes»), appartenait à la Compagnie de Jésus, et, selon Grandmaison, aurait porté le titre de prédicateur du Roi.

Texte

1 De la main de Pierre Picot.

2 L'ouvrage publié ne mentionne pas la composition du jury.

3 Ces deux ouvrages sont : l'Essai analytique sur les lois naturelles de l'ordre social (1800) et $\mathrm{Du}$ divorce considéré au dix-neuvième siècle (1801).

4 Pierre Picot a vraisemblablement évoqué le problème de la pluralité des mondes habités, et l'éventualité d'y rencontrer des créatures intelligentes. Buisson se réfère sans doute à Malebranche, Recherche de la vérité, Livre III, ch. V, VI, et surtout VII.

5 Jean-Etienne-François Marigné (1755-1830) chercha à défendre Louis XVI, émigra en Angleterre, où il traduisit les Mémoires de Gibbon (1797) et prépara une Vie de David Garrick (1801). Ami de J.-B.Suard, il le seconda dans l'édition du Publiciste. Il entretenait 
des liens d'amitié avec Louis de Bonald. Fontanes l'appela aux fonctions d'inspecteur général de l'Université. A ce titre, il fut en contact avec Marc-Auguste Pictet et chercha à seconder la création à Genève d'une école préparatoire de droit ainsi que d'une école préparatoire de médecine. Cf. Charles Borgeaud, Op. cit., tome II, pp. 197-201. Cf. l'article consacré à Marignié dans la Biographie universelle de Michaud, nouvelle éd., tome XXVI.

6 Le Publiciste, journal quotidien, parut entre le 7 nivôse an VI et le $1^{\text {er }}$ novembre 1810. Sur ce journal et ses collaborateurs, cf. Monglond A., La France révolutionnaire et impériale, tome VIII, col. 1315-1321.

7 Pierre Picot a dû sans doute s'enquérir sur les liens éventuels de son correspondant avec l'importante famille Buisson établie à Genève depuis plus de deux siècles. Cf. Galiffe J. A., Notices généalogiques sur les familles genevoises. Genève, Jullien, tome II, 1892. «Buisson», pp.511-520.

8 De la main de Pierre Picot. 


\section{Summary}

\section{Bichats cousin and the Geneva theologian}

A letter written in 1802 by M. F. R.Buisson to Pierre Picot

In a letter addressed to Pierre Picot, professor of divinity at the Academy of Geneva, the young doctor Matthieu-François-Régis Buisson (1776-1804) describes the climate of tension in which he had to defend his thesis at the Medical Faculty of Paris. He advocates a kind of holy alliance between Christian physicians and theologians against the party dominating the Ecole de médecine, in which the ideology of the Encyclopédie together with Pinel's anticlericalism and Cabanis's materialistic monism prevail.

\section{Zusammenfassung}

\section{Der "gläubige Arzt» und der Genfer Theologe}

Ein Brief aus dem Jahre 1802 von M. F. R. Buisson an Pierre Picot

In seinem Brief an den Genfer Theologieprofessor Pierre Picot berichtet der junge Arzt Matthieu-François-Régis Buisson (1776-1804) über das gespannte Klima, in welchem er an der Medizinischen Fakultät von Paris seine Dissertation verteidigen musste. Er empfiehlt eine Art heiliger Allianz der gläubigen Ärzte mit den Theologen gegen die an der Ecole de médecine herrschende Partei, wo die Ideologie der Encyclopédie, der Antiklerikalismus Pinels und der materialistische Monismus Cabanis' den Ton angeben.

Professeur Jean Starobinski

12, rue de Candolle

CH-1205 Genève 